\title{
A Survey on Removal of Duplicate Records in Database
}

\author{
M. Karthigha ${ }^{1 *}$ and S. Krishna Anand ${ }^{2}$ \\ 1 PG Student, School of Computing (CSE), SASTRA University, 613401, \\ Thanjavur, Tamilnadu, India; karthighamaniraj@gmail.com \\ 2 Senior Assistant Prof, School of Computing (CSE), SASTRA University, 613401, \\ Thanjavur,Tamilnadu, India; skanand86@gmail.com
}

\begin{abstract}
Deduplication is a task of identifying one or more records in repository that represents same object or entity. The problem is that the same data may be represented in different way in every database. While merging the databases, duplicates occur despite different schemas, writing styles or misspellings. They are called as replicas. Removing replicas from the repositories provides high quality information and saves processing time. This paper presents a thorough analysis of similarity metrics to identify similar fields in records and a set of algorithms and duplicate detection tools to detect and remove the replicas from the database.
\end{abstract}

Keywords: Similarity Metrics, Database, Indexing, Deduplication.

\section{Introduction}

Over a period of years, database plays a vital role in IT industries. These industries finally rely on the accuracy of databases to perform operations. When data are integrated from disparate sources to enforce data warehouse, the problem called data heterogeneity occurs. This is because of structure and semantics maintained in each and every database. Heterogeneity is also due to the variations in representation such as misspellings, typographical errors and abbreviations. Heterogeneity is of two types. They are structural heterogeneity and lexical heterogeneity. Each field in records maintain a unique structure for each database in structural heterogeneity. In lexical heterogeneity, all fields share similar structure, but they differ in manner of their representation. Data cleaning and standardization are performed to solve the heterogeneity problem.

The main objective of deduplication is to identify two or more records, which represents the same object. It was previously called as record matching and record linkage.
Replicas are called as dirty data $[4,9]$. Government agencies and companies have spent large amount of money to clean the dirty data in the repositories, which results in quality data content. Replica-free repositories will improve the efficiency and save the processing time. With increase in substantial amount of data, problems like security, low response time, quality assurance and availability begin to arise.

'Active learning' system was designed to identify the duplicates by creating disputing training pairs [1]. Then for record matching process, a framework called MARLIN was proposed [4]. Data standardization and various similarity metrics was utilized to find the matching between the strings in records [5].The above mentioned techniques do not deal with data security. Hence an Encryption scheme for deduplication was suggested [4].

Unsupervised duplicate detection algorithm explicates record matching over query results [9]. Later, several indexing techniques were discussed to minimize the time consumption [11]. An online deduplicator algorithm was

* Corresponding author:

M. Karthigha (karthighamaniraj@gmail.com) 
suggested to achieve higher performance, which minimizes the comparisons by forming clusters [10]. The above mentioned techniques cover field comparisons provided only by the user, which results in automated deduplication function $[8,12]$.An optimization technique called Particle Swarm Optimization was discussed [13]. KFINDMR algorithm was used to select the most represented samples to find the duplicates [16].

\subsection{Drawbacks of Using Dirty Data}

It has been observed during the years that there is a significant amount of time consumption owing to the large amount of additional data. This in turn leads to performance degradation. Besides, the presence of replicas also increases the operational costs. Moreover, the need for additional processing power significantly degrades the overall efficiency of the system. Hence, there is a dire need to establish a system to eliminate the duplicates.

\section{Study on Deduplication Techniques}

\subsection{Active Learning}

One of the duplicate detection systems named ALIAS was designed [1]. By the discovery of challenging training pairs, ALIAS promotes the automatic construction of deduplication function. The main difference between the ordinary and an active learner is that while the former one depends on a static training set, the latter actively picks subset of representations from an unlabeled data and it will be labelled, which provide more information to learner. The main role of ALIAS is to clearly distinguish between duplicate and non-duplicate pairs. ALIAS requires humans only when the uncertainty is very high to label the pair as duplicates or not. ALIAS does not sound to be a better approach, because it always requires some training data which is not available in real world [15].

\subsection{Marlin Framework}

A framework called MARLIN (Multiply Adaptive Record Linkage with Induction) was suggested for record matching process [4]. Marlin architecture consists of training phase and duplicate detection phase. In training phase, learnable distance metrics are trained for each field in record, which results in paired field duplicates and non-duplicates. Some individual fields which are not equivalent may also be pres- ent in duplicate records; hence it results in noisy training data, whilst it won't imply a serious problem. Duplicate detection phase acts by generating potential duplicate pairs. MARLIN framework employs clustering method to separate record into clusters, which consists of overlapping duplicates. Learned distance metrics are used to estimate the distance features of each field of records, producing distance features as input to the classifier. Classifier acquires the duplicates for each pair of records by using a measure called confidence. The problem lies in choosing a similarity threshold; hence it results in two similarity thresholds. One type indicates the high confidence duplicates and another indicates possible matches, which requires human expert to label.

\subsection{Identifying Duplicates by Data Preparation}

Identification of duplicates starts with data preparation stage, where fields (i.e. data) are stored in consistent manner in repository by solving the heterogeneity problem $[5$, 14]. Data preparation consists of parsing, data transformation and data standardization. The main goal of parsing is to locate, identify and isolate the data element from the source files. Parsing promotes the comparison of individual components which is used to match the data. Data transformation refers to data type conversion, element of one data type to another. It mainly focuses on one element at a time. Final step is data standardization, where data elements presented in various data formats are standardized to specific format. Due to standardization, data presented in various databases are converted into uniform representation. One of the best examples for data standardization is 'address'. Data standardization must be done before the record matching process starts. Many duplicates have been designated as non-duplicates, without standardization. After finishing the data preparation stage, data with their similar fields are stored in a table to find which field should be compared with another. Comparing the field "first name" and the field 'address' would not provide useful information to detect duplicates. However, other fields such as date, time, name and title pose standardization problems.

\subsection{Techniques to Measure the Similarity Between Individual Fields}

Various similarity metrics was proposed to determine the matches between the strings [5]. Most of the mismatches 
occur in database due to the typographical variations of string. Certain similarity metrics are used to handle inconsistencies occurred during typing. These metrics include token based similarity metrics, character based similarity metrics, phonetic similarity metrics and numeric similarity metrics.

\subsubsection{Character Based Similarity Metrics}

Character based similarity metrics covers typographical errors [5].

\subsubsection{Edit Distance}

Edit distance transforms one string into another by minimum number of edit operations. It can also be called as levenshtein distance. Edit operations are of three types. It can be done by either inserting or deleting or replacing a character by another character in the string.

\subsubsection{Affine Gap Distance}

Affine gap distance adds two edit operations namely opening and extending the gap. However, it fails to find the matches when the strings were shortened.

\subsubsection{Smith-Waterman Distance}

Smith waterman distance is called as substring matching. It identifies the matches in short distance by considering only the middle of the string, by ignoring the beginning and end of the strings. Distance between the strings can be computed by using Needleman and Wunsch algorithm. For example, Prof. 'Hillary R. Clinton, U.S' and Prof. 'Hillary R. Clinton' can be matched using Smith Waterman distance.

\subsubsection{Jaro Distance}

Jaro distance metric is used to compare first and last names. It identifies the common characters and the number of transpositions. Jaro metric assigns higher weight to prefix matches. This feature will play a pivotal role while considering matches in surnames.

\subsubsection{Token Based Similarity Metrics}

Token based similarity metrics identify similar strings sharing the same meaning by rearranging the strings [5].

\subsubsection{Q-grams with tf.idf}

Q-grams use tokens, instead of words. It identifies the matches despite the spelling error by means of inserting and deleting the words in the string. It also ignores low weight. For example, the string 'Gateway Communications' and another string 'Communication Gateway international' are matched under high similarity, because the word 'international' have low weight. This approach is more suitable for identifying the matches, though the words were misspelled.

\subsubsection{Atomic String}

Atomic string is the ratio of matched atomic strings to the mean of atomic strings. Atomic strings can be matched, if one string is a prefix of the other string or both the atomic strings are identical. Atomic string is a succession of alphanumerical characters, which were limited through punctuations.

\subsubsection{Phonetic Similarity Metrics}

Token and character based metric addresses the issue in representation of string. Strings which were not matched by using the above mentioned similarity metrics can be similar in this metric because of their phonetics. For example, the string Kageonne and the string Cajun are similar by using phonetics, though their representations are different [5].

\subsubsection{Soundex}

Soundex is the phonetic coding scheme which is used for matching surnames. It acts upon by grouping identical group of consonants which were phonetically similar.

\subsubsection{Metaphone and Double Metaphone}

Metaphone is an alternative for soundex. It uses 16 consonants to describe more number of words which were used in English and also in other than English words. By enhancing the metaphone, double metaphone was suggested, which allows numerous encodings for the names that, has several likely orthoepy.

\subsubsection{Oxford Name Compression Technique}

ONCA is used to group similar names by two steps. Initially, it performs compression. This is followed by the implementation of soundex algorithm with the resultant compressed data.

\subsubsection{Numeric Similarity Metrics}

Numeric similarity metric consider each number as string and the similarity can be identified using the metrics defined in the earlier section [5]. 


\subsection{Secure Data Deduplication}

Storer et al. [7] suggested an approach called secure data deduplication. The main idea behind the approach is to find the chunks in documents and the identified chunks are encrypted using keys. By matching the encrypted data, identical contents can be exploited. The drawback of this approach is that if the same content is encrypted using two different keys, it results in two different cipher texts, which makes it difficult to identify the duplicates. Hence it does not sound to be a feasible approach.

\subsection{Unsupervised Duplicate Detection}

An online record matching algorithm called Unsupervised Duplicate Detection (UDD) is used for web databases. UDD uses two types of classifiers namely Weighted Component Summing classifier (WCSS) and Support Vector Machine classifier (SVM). WCSS assigns weight to each field based on their distance (i.e.) by calculating the dissimilarity among the records. The main function of WCSS is to perform record matching. It identifies the record pairs from different data sources by adjusting the weights, which results in positive data set or duplicates and negative data set or non-duplicates. SVM classifier identifies the new duplicates from the given positive and negative data set. Finally, by using the resultant duplicates and non-duplicates, the field weights assigned in the first step can be adjusted to start new iteration again for identifying new duplicates. This iteration continues until no new duplicates are found. While the first classifier (WCSS) does not require any pre labelled training data, second classifier (SVM) requires limited training data [9].

\subsection{Indexing Techniques}

Several indexing techniques was suggested for record linkage, which uses blocking as a key technique [11]. Records are placed into their blocks using their Blocking Key Values $(\mathrm{BKV})$. By using BKV, records which were similar are inserted in one block and records that are dissimilar are placed in different block. Record linkage process consists of two phases namely build and retrieve.

\subsubsection{Build}

The process of building deals with the usage of an index or hash table during merging. Merging of the two databases could be done using both a separate index and data structure in every database or by using an individual data structure with shared key values.

\subsubsection{Retrieve}

Records that share the same bounded key values would be compared with another record in same block or in different database to generate candidate record pairs. Comparisons will be done by the classifier for the given record pairs to find the matches.

\subsubsection{Types of Indexing}

\subsubsection{Traditional Blocking}

Records that share the same keys are placed in one block and they were compared with another. The drawback in this approach is some records could be inserted into a wrong block and it is hard to forecast the entire count of record pair's generation [11].

\subsubsection{Sorted Neighbourhood Indexing}

Sorted array sorts the database based on bounded key values. It uses windows as key field. This approach influence records in current windows and pairs are generated from this window. The drawback in this approach is the choice of window size. A smaller value in this choice may lead to a case where it becomes physically impossible to compare all records sharing the same key [11].

\subsection{Q-gram}

Q-gram indexes the database by inserting the records which share the same bounded key value into alike block. Q-grams were implemented effectively in relational database management system [11].

\subsection{Suffix Array}

Suffix array can be obtained by inserting their bounded key value and its suffix into suffix array grounded inverted index. It is a collection of sequences or strings and their suffixes in alphabetical order. Its usage is widespread in bibliographic databases [11].

\subsection{Canopy Clustering}

Overlapping clusters are called as canopies and they are created using threshold or nearest neighbour mechanism. Candidate pairs are determined from overlapping clusters. Nearest neighbour approach results in large amount of true 
matches from the given record pairs. This method outperforms the threshold approach [11].

\subsection{String Map Based Indexing}

Each record is considered as a string and they are mapped onto multidimensional Euclidean space, followed by mapping into successive dimensional space. It uses $\mathrm{k}-\mathrm{d}$ tree to map the strings into multidimensional space for efficient matching [11]. Indexing techniques achieves minimum number of comparisons between records.

\subsection{Identification and Removal of Duplicated Records}

Bilal et.al. proposed a deduplicator algorithm to identify and remove the duplicate records [10] in 2011. In this approach, all the data values or fields are converted into numeric forms to create clusters. The fields can include parameters dealing with string and date. Initially, clusters are formed using $\mathrm{k}$ - means clustering algorithm to reduce the number of comparisons. Records within the clusters can be matched by using divide and conquer method. This sounds to be more efficient in terms of identifying fully duplicated records, partially duplicated records and erroneous duplicated records. This approach is more suitable for single tables, instead of multiple tables. Performance metric can be estimated by precision, recall and F-score.

\subsection{Genetic Programming Approach}

A Genetic Programming (GP) algorithm for deduplication was proposed $[8,12]$. The main factor of genetic programming is Evidence. Evidence is a combination of attribute and similarity functions, extracted from the given data content. By using evidence efficiently, GP generates an automated deduplication function to check whether two or more entries in a repository represent the same real world entity. The suggested deduplication function also adapts to a different identification boundary, based on the parameter. GP is found to have a better performance index as compared to MARLIN.

\subsection{Particle Swarm Optimization}

An optimization technique called Particle Swarm Optimization (PSO) algorithm was suggested for identifying and eliminating the duplicates [13]. The main idea behind the PSO algorithm is swarm. PSO algorithm includes two phases namely training and duplicate record detection phase. It uses cosine similarity and levenshtein distance to find the matches between the record pairs. The resultant data forms the feature vectors to represent the elements, which requires duplicate checking. Duplicate detection phase identifies the duplicates from the feature vectors by means of PSO algorithm. It outperforms genetic algorithm by providing high accuracy.

\subsection{Genetic Programming Using KFIND}

An algorithm called KFIND was suggested to enhance the accuracy of classifier to identify the duplicates [16]. KFIND improves the accuracy of the classifier by finding the most represented data samples. Distance is calculated by the mean value of the most represented data samples. If the minimum distance is less than the mean value, duplicates can be removed and hence it calculates the centroid for the new data set samples. These steps will be repeated until the required data samples are selected.

\section{Duplicate Record Detection Tools}

\subsection{FEBRL}

FEBRL stands for Freely Extensible Biomedical Record Linkage. It is available under open source software license to clean the data $[6,14]$. Several techniques were developed and encapsulated into Graphical User Interface (GUI), which performs data cleaning, data standardization, record linkage and deduplication. It allows both new and experienced users to learn more about record linkage techniques. It is more efficient to work with large number of data sets. Febrl comprises of two components namely data standardization and duplicate detection. While the former one depends on hidden Markov model, later one relies on similarity metrics such as q-gram and jaro metric to find the duplicates.

\subsection{TAILOR}

A record matching toolbox called TAILOR was suggested, which permits user to enforce dissimilar deduplication methods on various datasets $[2,14]$. It supports multiple models; hence it is also called as a flexible tool. Record linkage process in TAILOR consists of two steps. Initially, comparison vector is created for each field pair. Then 
matching status of record pairs is ascertained. Metrics such as performance and accuracy can also be compared with other models.

\subsection{Whirl}

Whirl is an open source duplicate detection system, which is obtained by combining cosine similarity and tf-idf weighting scheme $[5,14]$. Whirl uses token based similarity metric to find the similarity between the two strings in the lists. It can be used in academic and research studies.

\subsection{Big Match}

The role of big match is to perform deduplication between two types of files namely a large file A, and a moderate file $B$. The process works by choosing the records from $A$ that corresponds to file B. Several parameters such as matching and blocking field can be specified by the user in the big match program. For each blocking criterion, a key list is created by reading the file $\mathrm{B}$, and then it moves to $\mathrm{A}$. The program finds the matches between the files through their same key value and it also computes the comparison weight. This program continues by reading the next records from file A [3].

\section{Conclusion}

In this paper a detailed analysis of subsisting methodologies, which were used for record matching and deduplication are confronted. It has been estimated from the above techniques that deduplication is a cumbersome process and requires both time and memory. An effective and efficient deduplication algorithm, which requires minimum number of comparisons for records with less memory and time need to be developed in future.

\section{References}

1. Sarawagi S, and Bhamidipaty A (2002). Interactive deduplication using active learning, Proceedings of the eighth ACM SIGKDD International 1 Conference. Knowledge Discovery and Data Mining (KDD '02), 269-278.

2. Elfeky M G, Elmagarmid A K et al. (2002). Proceedings of the International Conference on Data Engineering. (ICDE '02), $17-28$.
3. Yancey W E (2002). Bigmatch: A program for extracting probable matches from a large file for record linkage, Technical Report Statistical Research Report Series RRC2002/01, US Bureau of the Census, Washington, D.C.

4. Bilenko M, and Mooney R J (2003). Adaptive duplicate detection using learnable string similarity measures, KDD '03 Proceedings of the ninth ACM SIGKDD international conference on Knowledge discovery and data mining, 39-48.

5. Elmagarmid A K, Ipeirotis P G et al. (2007). Duplicate record detection: a survey, IEEE Transactions on Knowledge and Data Engineering, vol 19(1), 1-16.

6. Freely extensible biomedical record linkage, 2007. Available from http://sourceforge.net/projects/febrl, vol 2011, no. 1, 1-16.

7. Storer M W, Greenan K et al. (2008). Secure data deduplication, Storage SS ' 08 Proceedings of the 4th ACM international workshop on Storage security and survivability, 1-10.

8. deCarvalho M G, Laender A H F et al. (2008). Replica Identification Using Genetic Programming, SAC '08 Proceedings of the 2008 ACM symposium on Applied computing, 1801-1806.

9. Wang W J, Lochovsky F H (2010). Record matching over query results from multiple web databases, IEEE Transactions on Knowledge and Data Engineering, vol 22(4), 578-588.

10. Khan B, Rauf A et al. (2011). Identification and removal of duplicated records, World Applied Sciences Journal, vol 13(5), 1178-1184.

11. Christen P (2011). A survey of indexing techniques for scalable record linkage and deduplication, IEEE Transactions on Knowledge and Data Engineering, vol 24(9), 1537-1555.

12. deCarvalho M G, Alberto H F et al. (2013). A genetic programming approach to record deduplication, IEEE Transactions on Knowledge and Data Engineering, vol 24(3), 399-412.

13. Deepa K, and Rangarajan R (2012). Record deduplication using particle swarm optimization, European Journal of Scientific Research, vol 80(3), 366-378.

14. Subramaniya swamy V, and Pandian S C (2012). A complete survey of duplicate record detection using data mining techniques, Information Technology Journal, vol 11(8), 941-945.

15. Banu A F, and Chandrasekar C (2012). A survey on deduplication methods, International Journal of Computer Trends and Technology, vol 3(3), 364-368.

16. Shanmugavadivu P, and Baskar N (2012). An improved genetic programming based approach to deduplication using KFINDMR, International Journal of Computer Trends and Technology, vol 3(5), 694-701. 